\title{
Prognostic Value of Arterial/Alveolar Oxygen Tension Ratio (a/APO2) in Acute Pulmonary Embolism
}

\author{
Jen Te Hsu, MD; Chi Ming Chu, PhD*; Shih Tai Chang, MD; Hui Wen Cheng, MD; \\ Pi Chi Lin, MD; Tsu Shiu Hsu, MD**; Ju Feng Hsiao, MD**; \\ Wan Ching Ho, MD**; Chang Min Chung, MD
}

\begin{abstract}
Background Because the arterial/alveolar oxygen tension ratio (a/APO2) is relatively constant throughout the entire range of fractional inspired oxygen concentration ( $\mathrm{FiO} 2)$, its use in determining the prognosis of acute pulmonary embolism (APE) was investigated.

Methods and Results This study retrospectively assessed 202 consecutive patients with APE confirmed by computed tomography or high probability lung scintigraphy. All patients underwent initial arterial blood gas analysis during the first $24 \mathrm{~h}$ of admission. Receiver-operating characteristic analyses were performed to determine the a/APO2 cut-off value for predicting 30-day death or 30-day composite events. Cut-off values for a/APO2 were used to determine stability in all patients and 2 subgroups $(0.49$ for all patients; 0.49 for FiO2= $0.21 ; 0.46$ for $\mathrm{FiO} 2>0.21$ ). Using the cut-off value of a/APO2 $<0.49$ for predicting 30 -day death, the negative predictive value (NPV) was $90 \%$, and the positive predictive value (PPV) was 30.3\%. For the 30-day composite end point, the NPV was $81.3 \%$, and the PPV was 40.9\%. Excluding massive APE, the a/APO2 also had high NPV and moderate PPV in predicting short-term prognosis. This study additionally demonstrated a linear relationship between platelet count and a/APO2.
\end{abstract}

Conclusions The cut-off value of a/APO2 $<0.49$ exhibits stability at variable FiO2 values and is a useful prognostic predictor in APE. (Circ J 2007; 71: 1560-1566)

Key Words: Acute pulmonary embolism; Alveolar-arterial oxygen pressure difference (AaDO2); Arterial/alveolar oxygen tension ratio (a/APO2); Thrombocytopenia

$\mathbf{S}$ hort-term mortality is high in patients with a pulmonary embolism (PE). The International Cooperative Embolism Registry (ICOPER) of 2,454 patients reported that the short-term cumulative mortality because of PE was $11.4 \%$ at 2 weeks and $17.4 \%$ at 3 months! Acquired predisposing factors include surgery, trauma, immobilization, cancer, oral contraceptive use, pregnancy, postmenopausal hormone replacement therapy and frequent air travel? Accurate risk stratification is of paramount importance in selecting the optimal management strategy for patients with acute PE (APE) and a rapid, noninvasive and inexpensive screening test is urgently needed. Although arterial blood gas $(\mathrm{ABG})$ measurements have proved disappointing for excluding $\mathrm{APE}$, both the alveolar-arterial oxygen gradient $(\mathrm{AaDO} 2)$ and the arterial/alveolar oxygen tension ratio (a/APO2) have demonstrated efficacy for prognosis of APE4 The previous study by Hsu et al was limited to patients breathing room air4 Although AaDO2 is believed to measure the severity of APE, it is strongly

(Received April 3, 2007; revised manuscript received June 13, 2007; accepted June 18, 2007)

Division of Cardiology, Chiayi Chang Gung Memorial Hospital, Chang Gung University College of Medicine, *Section of Health Informatics, Institute of Public Health, National Defense Medical Center and University and **First Division, Cardiovascular Section, Lin-Kou Medical Center, Chang-Gung Memorial Hospital, Taipei, Taiwan

Mailing address: Chang Min Chung, MD, Division of Cardiology, Chiayi Chang Gung Memorial Hospital, 6, Sec. West Chai-Pu Road, $\mathrm{Pu}-\mathrm{Tz}$ City, Chai Yi Hsien, Taiwan. E-mail: cmchung02@ hotmail. com dependent on the fraction of inspired oxygen $(\mathrm{FiO} 2)^{5,6}$ The a/APO2 has proven in several studies to be a more reliable predictive index in patients with abnormal lung gas exchange ${ }^{5-7}$

Therefore, the purpose of the present investigation was to use an $\mathrm{FiO} 2$ expanded from 0.21 to 1.0 to explore further the prognostic value of a/APO2.

\section{Methods}

\section{Study Sample}

We assessed 202 consecutive patients admitted to Chang Gung Memorial Hospital (CGMH), Taiwan, between March 2000, and July 2005, with APE confirmed by computed tomography (CT) or high-probability ventilationperfusion lung scan. During the first $24 \mathrm{~h}$ of admission, all enrolled patients underwent initial ABG analysis under either room air or oxygen support. Patients with an intermediate- or low-probability lung scan, clinical suspicion of septic emboli, recurrent PE or history of chronic lung disease were excluded.

APE was treated pharmacologically in all cases; that is, anticoagulant therapy with unfractionated heparin dosed according to activated partial thromboplastin time, or weightadjusted low-molecular heparin administered subcutaneously. Thrombolysis, which 16 patients underwent, consisted of a 2-h intravenous infusion of $100 \mathrm{mg}$ recombinant tissue plasmin activator (tPA) without concomitant heparin.

Oral warfarin at an international normalized ratio of 2.03.0 was used for at least 6 months in all discharged patients. The Human Research Committee approved the study and 
Table 1 Clinical Characteristics, Arterial Blood Gas Analysis, Echocardiographic Parameters and Cardiac TnI Levels in Patients Who Survived 30 Days or Died 30 Days After Acute Pulmonary Embolism

\begin{tabular}{|c|c|c|c|}
\hline & 30-day survival $(n=157)$ & 30-day death $(n=45)$ & $p$ value \\
\hline \multicolumn{4}{|l|}{ Clinical characteristics } \\
\hline Age (years) & $62.80 \pm 15.68$ & $62.42 \pm 17.40$ & $0.891 *$ \\
\hline Duration of symptoms (days) & $6.33 \pm 8.29$ & $6.80 \pm 10.65$ & $0.755 *$ \\
\hline Women & $81(51 \%)$ & $23(51 \%)$ & $1.000 * *$ \\
\hline Cancer & $21(13 \%)$ & $19(42 \%)$ & $<0.001 * *$ \\
\hline$S B P$ & $131.29 \pm 27.71$ & $116.49 \pm 27.21$ & $0.002 *$ \\
\hline Shock $(S B P<90 \mathrm{mmHg})$ & $8(5 \%)$ & $11(24 \%)$ & $<0.001 * *$ \\
\hline Diabetes mellitus & $28(18 \%)$ & $9(20 \%)$ & $0.827 * *$ \\
\hline Hypertension & $44(28 \%)$ & $8(18 \%)$ & $0.182 * *$ \\
\hline Congestive heart failure & $12(8 \%)$ & $2(4 \%)$ & $0.740 * *$ \\
\hline Deep vein thrombosis & $53(34 \%)$ & $12(27 \%)$ & $0.470 * *$ \\
\hline Coronary artery disease & $7(4 \%)$ & $1(2 \%)$ & $0.687 * *$ \\
\hline Renal insufficiency & $22(14 \%)$ & $7(16 \%)$ & $0.811 * *$ \\
\hline Recent surgery/immobilization & $18(11 \%)$ & $5(11 \%)$ & $1.000 * *$ \\
\hline Thrombocytopenia & $35(22 \%)$ & $22(49 \%)$ & $0.001 * *$ \\
\hline \multicolumn{4}{|l|}{ Arterial blood gas analysis } \\
\hline $\mathrm{FiO} 2$ & $0.28 \pm 0.13$ & $0.34 \pm 0.20$ & $0.023 *$ \\
\hline $\mathrm{PaO} 2$ & $69.25 \pm 40.02$ & $59.87 \pm 26.74$ & $0.141 *$ \\
\hline $\mathrm{PaCO} 2$ & $31.20 \pm 6.92$ & $30.05 \pm 10.49$ & $0.390 *$ \\
\hline $\mathrm{AaDO} 2$ & $94.38 \pm 70.68$ & $146.09 \pm 132.47$ & $0.001 *$ \\
\hline a/APO2 & $0.46 \pm 0.15$ & $0.36 \pm 0.16$ & $<0.001 *$ \\
\hline$a / A P O 2<0.49$ & $85(54 \%)$ & $37(82 \%)$ & $<0.001 * *$ \\
\hline $\mathrm{PaO} 2 / \mathrm{FiO} 2$ & $252.26 \pm 79.93$ & $205.60 \pm 90.74$ & $0.001 *$ \\
\hline \multicolumn{4}{|l|}{ Thrombolysis } \\
\hline Tissue plasmin activator & $12(8 \%)$ & $4(9 \%)$ & $0.759 * *$ \\
\hline \multicolumn{4}{|l|}{ Echocardiography and TnI } \\
\hline$R V D(R V / L V \geq 1)$ & $31(20 \%)$ & $17(38 \%)$ & $0.017 * *$ \\
\hline$T n I \geq 0.4 \mathrm{ng} / \mathrm{ml}$ & $86(55 \%)$ & $34(75 \%)$ & $<0.001 * *$ \\
\hline
\end{tabular}

TnI, troponin I; SBP, systolic blood pressure; RVD, right ventricular dilatation; RV, right ventricle; LV, left ventricle. See text for definitions of arterial blood gas analysis.

*Student's t-test, **proportional test.

all participants gave informed consent.

\section{Clinical Features and Biochemical Data}

Recorded clinical data including the following: age, gender, duration of symptoms, underlying disease and possible risk factors. Baseline biochemical data, such as blood urea nitrogen, serum creatinine, troponin I (TnI), ABG and platelet count were also examined before treatment. Electrocardiography, chest X-ray and echocardiographic findings were also reviewed.

The $\mathrm{AaDO} 2$ was calculated as:

$\mathrm{AaDO} 2(\mathrm{mmHg})=\mathrm{PAO} 2-\mathrm{PaO} 2$

$\mathrm{PAO} 2=(\mathrm{PB}-\mathrm{PH} 2 \mathrm{O})(\mathrm{FiO} 2)-1.25 \mathrm{PaCO} 2$

and the a/APO2 as:

$\mathrm{a} / \mathrm{APO} 2=\mathrm{PaO} 2 / \mathrm{PAO} 2$

where $\mathrm{Pв}_{\mathrm{B}}$ is barometric pressure (assuming 760 torr), Рн2о is the water vapor partial pressure $\left(47\right.$ torr at $\left.37^{\circ} \mathrm{C}\right), \mathrm{FiO} 2$ is fractional inspired oxygen concentration, $\mathrm{PaCO} 2$ is partial pressure of carbon dioxide in arterial blood $(\mathrm{mmHg}), \mathrm{PaO} 2$ is partial pressure of oxygen in arterial blood $(\mathrm{mmHg})$ and PAO2 is partial pressure of oxygen in alveolar. ABGs were measured while the patient breathed room air or was on oxygen support. All measurements were obtained within $24 \mathrm{~h}$ prior to anticoagulant therapy.

\section{Clinical Endpoints}

The 30-day all-cause death was the primary endpoint. The secondary endpoint was a composite endpoint of 30-day death and in-hospital complications, including cardiopulmonary resuscitation, mechanical ventilation and vasopressors for systemic arterial hypotension. Because this study was a retrospective observational study, the decision to start thrombolysis was based on the clinical assessment of the individual patient by the physician in charge. To minimize selection bias, thrombolysis was not included in the composite endpoint. All enrolled patients received followup treatment for at least 1 year.

\section{Statistical Analysis}

Continuous variables were compared by Student's t-test, and categorical variables between groups were compared by proportion test according to primary and secondary endpoints.

Receiver-operating characteristic (ROC) analyses was used to determine the a/APO2 cut-off value for predicting primary and composite endpoints. The reciprocal AaDO2 (AaDO2-R) was reciprocal for $\mathrm{AaDO} 2$, which equaled 1/AaDO2. The AaDO2-R was used to demonstrate the mirror curve of AaDO2 at the opposite side of ROC analyses. This analysis was used to compare the predictive power of different ROC curves of $\mathrm{AaDO} 2$ and a/APO2.

Cumulative probability of the primary and composite endpoints in patients with and without decreased a/APO2 were estimated by multivariate Cox regression analysis. The hazard ratio (HR) of a/APO2 was calculated by the Cox proportional hazard model to predict primary and composite endpoints. Multivariate analysis was then performed to identify predictors for 30-day death using the proportional hazards model with $95 \%$ confidence intervals (CI).

The strength of linear relationships between platelet count and a/APO2 was estimated on the basis of Pearson correlation coefficients. 
Table 2 Clinical Characteristics, Arterial Blood Gas Analysis, Echocardiographic Parameters and Cardiac Troponin I in the 30-Day Composite-Event-Free Group vs 30-Day Composite-Event Group

\begin{tabular}{lccc}
\hline \hline & $\begin{array}{c}\text { 30-day free of composite } \\
\text { endpoint }(n=137)\end{array}$ & $\begin{array}{c}\text { 30-day composite } \\
\text { endpoint }(n=65)\end{array}$ & p value \\
\hline Clinical characteristics & & & \\
Age (years) & $62.70 \pm 15.70$ & $62.74 \pm 16.83$ & $0.988^{*}$ \\
Duration of symptoms (days) & $6.05 \pm 8.05$ & $7.25 \pm 10.35$ & $0.371^{*}$ \\
Women & $65(47 \%)$ & $39(60 \%)$ & $0.100^{* *}$ \\
Cancer & $18(13 \%)$ & $22(34 \%)$ & $0.001^{* *}$ \\
SBP & $132.69 \pm 28.36$ & $118.09 \pm 25.31$ & $0.001^{*}$ \\
Shock (SBP $<90$ mmHg) & $7(5 \%)$ & $12(18 \%)$ & $0.002^{* *}$ \\
Diabetes mellitus & $23(17 \%)$ & $14(22 \%)$ & $0.440^{* *}$ \\
Hypertension & $37(27 \%)$ & $15(23 \%)$ & $0.608^{* *}$ \\
Congestive heart failure & $9(7 \%)$ & $5(8 \%)$ & $0.772^{* *}$ \\
Deep vein thrombosis & $49(36 \%)$ & $16(25 \%)$ & $0.147^{* *}$ \\
Coronary artery disease & $6(4 \%)$ & $2(3 \%)$ & $1.000^{* *}$ \\
Renal insufficiency & $16(12 \%)$ & $13(20 \%)$ & $0.134^{* *}$ \\
Recent surgery/immobilization & $15(11 \%)$ & $8(12 \%)$ & $0.814^{* *}$ \\
Thrombocytopenia & $28(20 \%)$ & $29(45 \%)$ & $<0.001^{* *}$ \\
Arterial blood gas analysis & & & $0.006^{*}$ \\
FiO2 & $0.28 \pm 0.12$ & $0.34 \pm 0.19$ & $0.587^{*}$ \\
PaO2 & $68.16 \pm 27.65$ & $65.07 \pm 53.03$ & $0.362^{*}$ \\
PaCO2 & $31.29 \pm 7.10$ & $30.21 \pm 9.23$ & $<0.001^{*}$ \\
AaDO2 & $90.44 \pm 68.91$ & $138.49 \pm 118.40$ & $<0.001^{*}$ \\
a/APO2 & $0.48 \pm 0.14$ & $0.36 \pm 0.17$ & $<0.001^{* *}$ \\
a/APO2 $<0.49$ & $72(53 \%)$ & $50(77 \%)$ & $<0.001^{*}$ \\
PaO2/FiO2 & $256.67 \pm 74.89$ & $210.65 \pm 95.10$ & $0.048^{* *}$ \\
Thrombolysis & & & $0.013^{* *}$ \\
Tissue plasmin activator & $7(5 \%)$ & $9(14 \%)$ & $<.001^{* *}$ \\
Echocardiography and troponin I & & & \\
RVD RV/LV $\geq 1)$ & $25(18 \%)$ & $23(35 \%)$ & $47(72 \%)$ \\
TnI $\geq 0.4$ ng/ml & $73(53 \%)$ & & \\
\hline Abbrevions see in Table & & & \\
\end{tabular}

Abbreviations see in Table 1.

*Student's t-test, **proportional test.

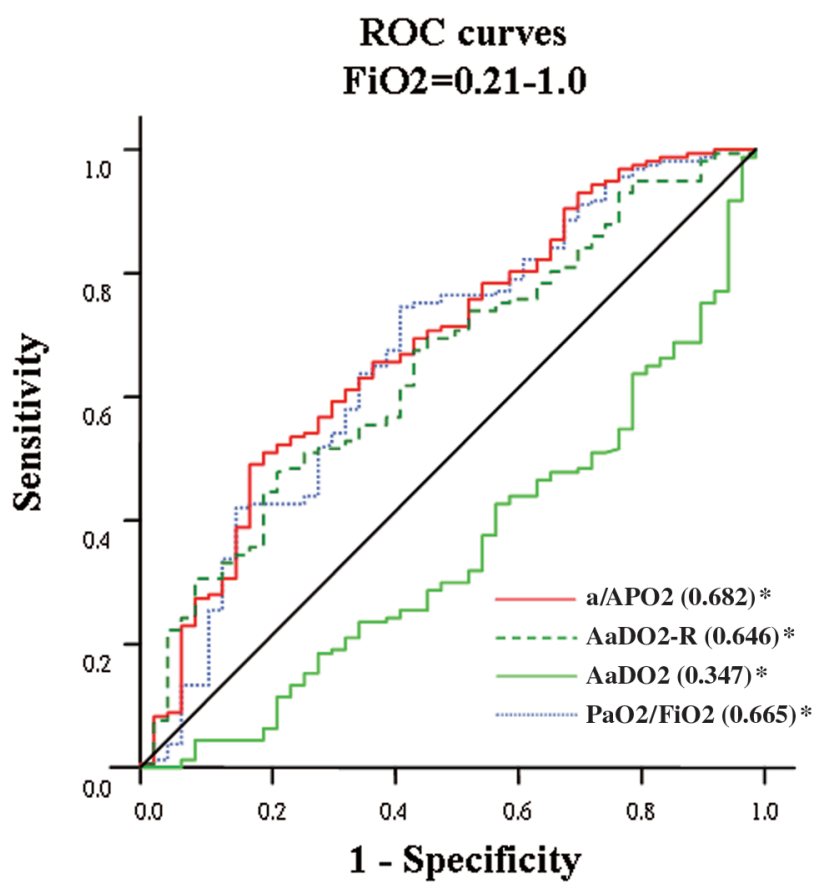

Fig 1. Receiver-operating characteristic (ROC) analysis curves for variable arterial blood gas analysis in all patients. *Area under the ROC curve. a/APO2, arterial/alveolar oxygen tension ratio; AaDO2-R, reciprocat the alveolar-arterial oxygen gradient; AaDO2, alveolararterial oxygen gradient; FiO2, fractional inspired oxygen concentration.

\section{Results}

Tables 1 and 2 show the baseline characteristics of the study population of 202 patients according to the primary and secondary endpoints, respectively.

In the 202 patients, diagnosis of PE was based on a highprobability lung scan in $121(60 \%)$ and a positive CT scan in $81(40 \%)$. The 30 -day mortality rate was $22.3 \%$, and the 30-day composite event rate was $32.2 \%$. The clinical parameters that differed significantly between the 30-day death group and the 30-day survival group included cancer, systolic blood pressure (SBP), shock ( $\mathrm{SBP}<90 \mathrm{mmHg}$ ), thrombocytopenia, FiO2, $\mathrm{AaDO} 2, \mathrm{a} / \mathrm{APO} 2, \mathrm{PaO} 2 / \mathrm{FiO} 2$, right ventricular (RV) dilatation and elevated TnI level. The only additional significant parameter between the 30day composite event group and the 30-day event-free survival was the rate of thrombolysis. In the 30-day composite event group, the rate of tPA treatment was higher than in the 30-day composite-event-free group. In ABG analysis, $\mathrm{FiO} 2$ was higher in the 30-day death group than in the 30day survival group $(p=0.023)$. Moreover, the FiO2 was also higher in the 30-day composite-event group than in the 30day event-free group $(\mathrm{p}=0.006)$.

This study used ROC curves to demonstrate differences in ABG variables. All patients $(n=202)$ were assigned to 1 of 2 subgroups according to $\mathrm{FiO} 2$ level. The first subgroup included patients breathing room air $(\mathrm{FiO} 2=0.21, \mathrm{n}=114)$, and the second subgroup included patients breathing a higher oxygen supplement ( $\mathrm{FiO} 2$ ranging from 0.28 to 1.0, $\mathrm{n}=88$ ).

Optimal cut-off values of a/APO2 and AaDO2 were calculated in all patients and the separate subgroups (Figs 1,2). 

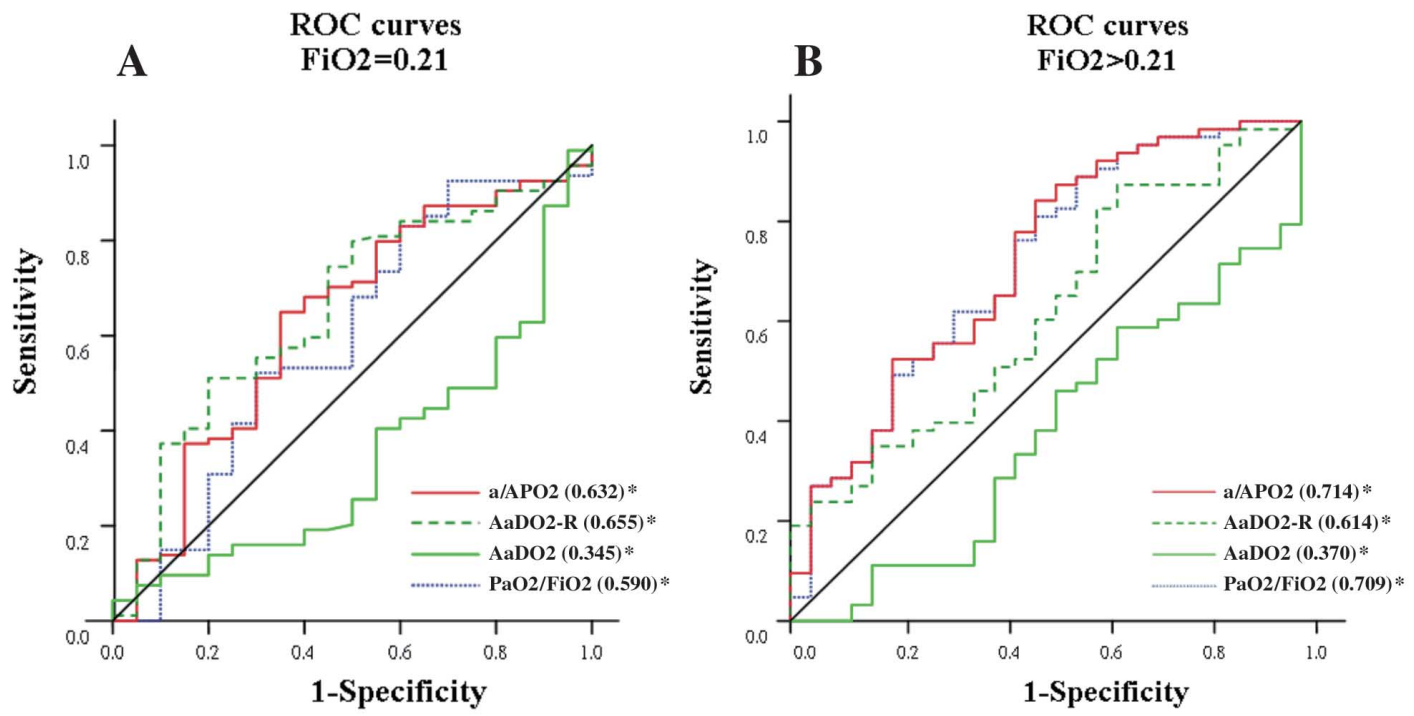

Fig 2. Receiver-operating characteristic (ROC) analysis curves for variable arterial blood gas analysis in 2 subgroups: (A) fractional inspired oxygen concentration $(\mathrm{FiO} 2)=0.21$, (B) FiO2 $>0.21$. *Area under the ROC curve. a/APO2, arterial/alveolar oxygen tension ratio; $\mathrm{AaDO} 2-\mathrm{R}$, reciprocat the alveolar-arterial oxygen gradient; $\mathrm{AaDO} 2$, alveolar-arterial oxygen gradient.

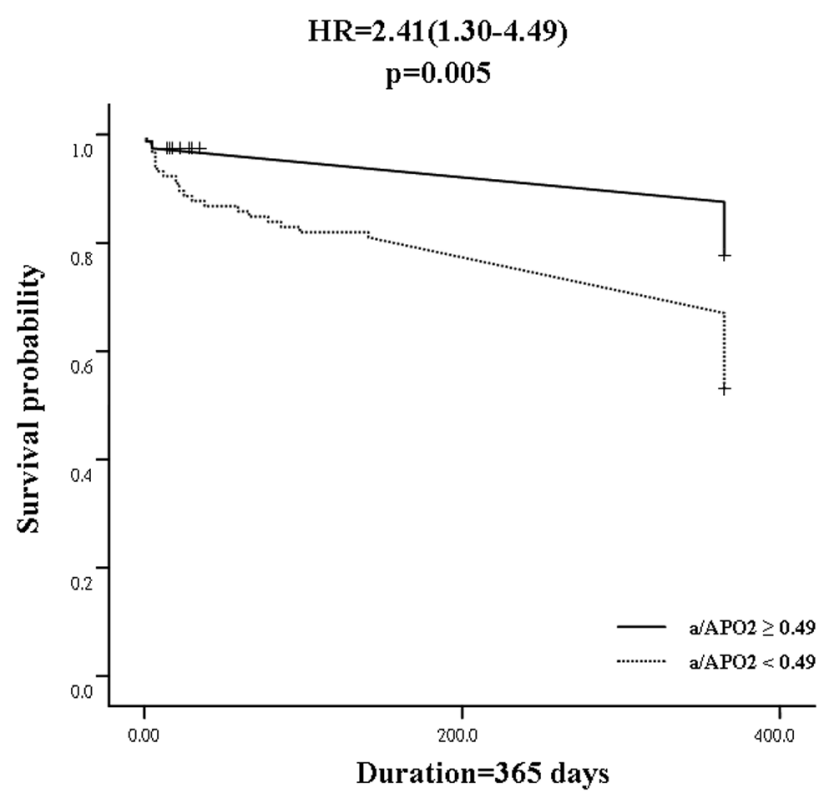

Fig 3. Survival curves at 1 year based on cut-off value of arterial/alveolar oxygen tension ratio $(\mathrm{a} / \mathrm{APO} 2)<0.49$ vs a/APO2 $\geq 0.49$. HR, hazard ratio.

Cut-off values of a/APO2 were stable in the total patient cohort and in the 2 subgroups. (0.49 for all patients; 0.49 for $\mathrm{FiO} 2=0.21 ; 0.46$ for $\mathrm{FiO} 2>0.21$ ); the optimal cut-off values of $\mathrm{AaDO} 2$ were highly variable in the total group and the 2 subgroups $(135 \mathrm{mmHg}$ for all patients; $53 \mathrm{mmHg}$ for $\mathrm{FiO} 2=0.21 ; 207 \mathrm{mmHg}$ for $\mathrm{FiO} 2>0.21$ ).

Fig 1 shows the differences in ABG variables in all patients. AaDO2-R instead of $\mathrm{AaDO} 2$ was used to compare the prognostic effect of $\mathrm{AaDO} 2$ and a/APO2. The area under the ROC curve for a/APO2 was higher than that for AaDO2-R (0.682 vs 0.646$)$. The ROC curves indicated that a/APO2 was the best predictor of short-term mortality. The optimal cut-off value for a/APO2 was 0.49 . Using the cut-
$\mathrm{HR}=2.45(1.37-4.36)$

$\mathrm{p}=0.002$

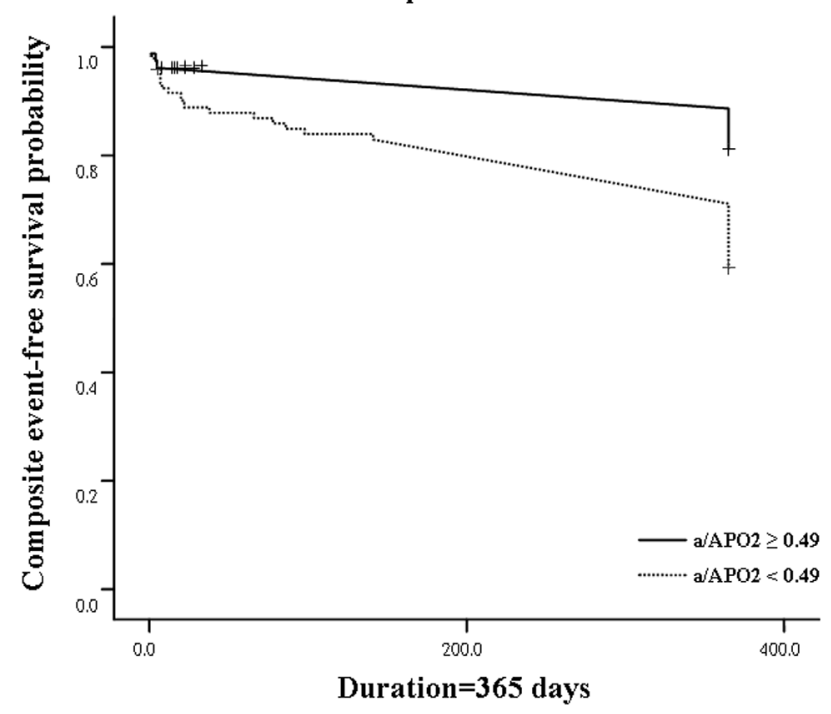

Fig 4. Composite event-free survival curves at 1 year based on cutoff values of arterial/alveolar oxygen tension ratio $(\mathrm{a} / \mathrm{APO} 2)<0.49 \mathrm{vs}$ a/APO2 $\geq 0.49$. HR, hazard ratio.

off value to distinguish 30 -day mortality and survival, the incidence of a/APO $2<0.49$ also revealed a significant difference between the 2 groups $(\mathrm{p}<0.001$, Table 1$)$. The incidence of a/APO $2<0.49$ also differed significantly between the 30-day composite event group and the event-free group $(\mathrm{p}<0.001$, Table 2$)$.

The cut-off value of a/APO2 $<0.49$ used to predict 30 day death demonstrated a sensitivity of $82 \%$ and a specificity of $45.8 \%$. The negative predictive value (NPV) was $90 \%$, and the positive predictive value (PPV) was $30.3 \%$. The 30 -day composite endpoint, a/APO2 $<0.49$, demonstrated a sensitivity of $76.9 \%$ and a specificity of $47.4 \%$. The NPV was $81.3 \%$, and the PPV was $40.9 \%$. 


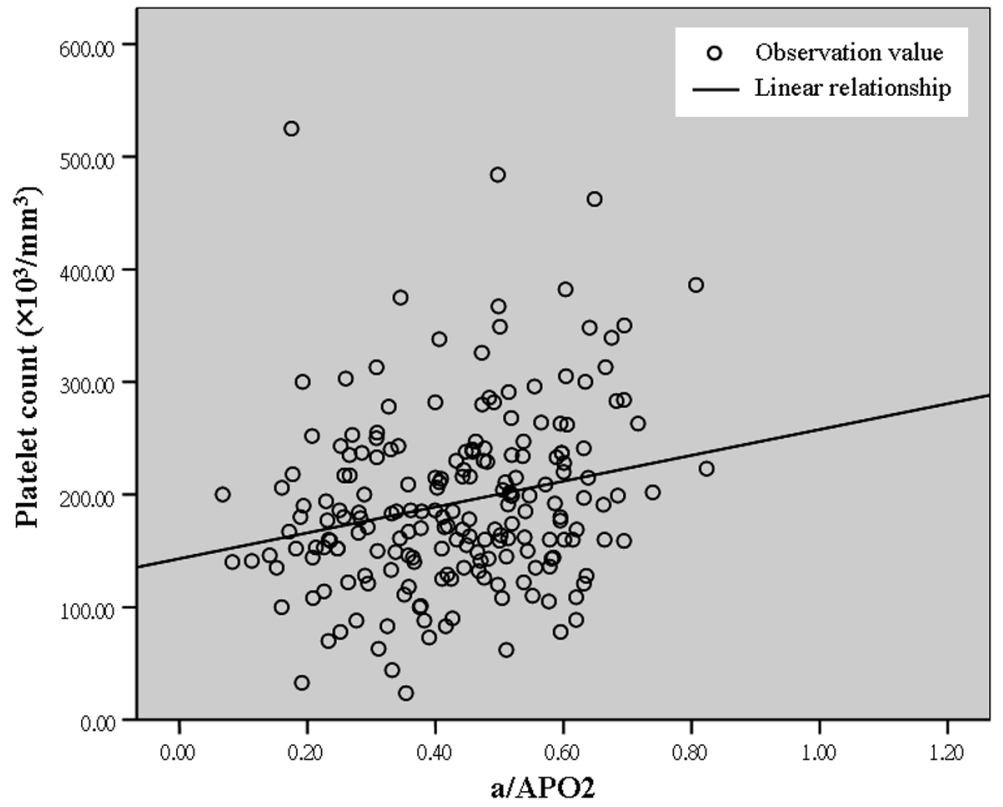

Fig 5. Platelet count as a function of arterial/alveolar oxygen tension ratio (a/APO2) in patients with acute pulmonary embolism. Correlation coefficient $(r)=0.233$, $\mathrm{p}<0.01$.
Patients in shock were classified as massive PE and excluded from the normotensive subgroup analysis $(n=19)$. Using the cut-off value (a/APO2 <0.49) to analyze normotensive APE $(n=183)$, the cut-off value for 30-day death had a sensitivity of $85.3 \%$ and a specificity of $48.3 \%$. The NPV was $93.5 \%$, and the PPV was $27.3 \%$. For the 30 -day composite endpoint, a/APO $2<0.49$ had a sensitivity of $77.3 \%$ and a specificity of $50.0 \%$. The NPV was $84.4 \%$, and the PPV was $38.7 \%$

Figs 3 and 4 show the short-term and 1-year survival curves for mortality and composite events, respectively. These survival curves also demonstrated a significant difference between 30-day mortality vs survival, as well as for 30-day composite-event vs event-free, based on the cut-off value for a/APO2 after adjusting for other significant interfering factors and age. For 30-day death, the HR of a/APO2 $<0.49$ was 2.17 (95\% CI 1.25-3.77); for the 30-day composite event, the HR of a/APO2 $<0.49$ was $2.19(95 \% \mathrm{CI}$ $1.32-3.62)$.

In addition, the incidence of thrombocytopenia significantly differed between both 30-day mortality vs survival and 30-day composite-event vs event-free. The odds ratio for 30-day mortality was 1.52 (95\% CI 1.17-1.97). The odds ratio for 30-day composite endpoint was 2.25 (95\% CI 1.38-3.68). A linear relationship between platelet count and a/APO2 level was also noted (Fig 5). Linear regression analysis revealed regression coefficients of 0.233 between platelets and a/APO2 $(\mathrm{p}<0.01)$.

\section{Discussion}

\section{Mechanisms of Impaired Gas Exchange and Index of Gas Exchange in APE}

Large shunt has proved to be the major cause of hypoxemia and impaired gas exchange in APE 8,9 An increased area of pulmonary artery obstruction destabilizes normal distribution of ventilation perfusion and increases shunt. The lung regions exhibit alveolar dead space with high ventilation/perfusion ratios and shunting because of perfusion of atelectatic areas? Other proposed mechanisms have been reported and revealed minor roles ${ }^{10-13}$
Several parameters are derived from ABG analysis. Hypoxemia and impaired gas exchange indices calculated as a function of inspired oxygen concentration include $\mathrm{PaO} 2 / \mathrm{FiO} 2, \mathrm{a} / \mathrm{APO} 2$ and AaDO25,13,14 In the present study, ROC curve analysis found that $\mathrm{PaO} 2 / \mathrm{FiO} 2$ was inferior to both the a/APO2 and AaDO2 for comparing gas exchange under room air. If $\mathrm{FiO} 2$ was more than 0.21 , the area under the ROC curve for $\mathrm{PaO} 2 / \mathrm{FiO} 2$ was slightly smaller than that for a/APO2 (0.709 vs 0.714). this finding was compatible with the report of Gowda et al. ${ }^{15}$ In addition, the unit of $\mathrm{PaO} 2 / \mathrm{FiO} 2$ was confusing and did not account for different levels of $\mathrm{PaCO} 2$. Because this, the $\mathrm{PaO} 2 / \mathrm{FiO} 2$ was not favorable for predicting the short-term prognosis of APE.

Although AaDO2 has been correlated with perfusion defects, including acute and chronic PE 14 AaDO2 would increase as FiO2 increased. Compared with AaDO2, a/APO2 was more consistent for identifying lung gas exchange under differing $\mathrm{FiO}_{2}$ - $^{-7}$

\section{Role of a/APO2 in APE}

In 1974, Gilberts and Keighley demonstrated that a/APO2 was more stable than AaDO2 at various levels of FiO25. The lower limits of normal were determined to be 0.75 . Their method was based on demonstrating that the ratio of arterial to alveolar oxygen tension is a reliable and consistent index of respiratory impairment over the range of $\mathrm{FiO} 2$ from 0.21 to 1.0.

Subsequent studies by Gilberts et al proved that the constancy of the a/APO2 ratio depends on the amount of shunting and ventilation/perfusion inequality? It was found that a/APO2 was most useful at FiO2 levels greater that 0.3 and $\mathrm{PaO} 2$ levels less that 100 torr. The greatest constancy of a/APO2 was noted in patients with large shunts, whereas AaDO2 shows the greatest variability under these circumstances.

In the emergency room, APE patients present with variable acuity and require different ventilation methods and oxygen treatment. Although a/APO2 is not independent of $\mathrm{FiO} 2$, it is clearly less dependent on $\mathrm{FiO} 2$ than $\mathrm{AaDO} 2$, and may be useful on this basis alone.

The present study also demonstrated consistent cut-off 
values of a/APO2 at different FiO2 levels. Conversely, the $\mathrm{AaDO} 2$ cut-off values were highly variable at different $\mathrm{FiO} 2$ levels, which indicates that a/APO2 is best for assessing the prognosis of APE because of its stability over a wide range of inspired oxygen concentrations.

\section{Role of a/APO2 in APE in Normotensive Patient}

Many reports has used echocardiography for risk stratification of in-hospital death and patients with echocardiographic evidence of RV dysfunction have a 4.6-12.6\% PErelated in-hospital mortality rate ${ }^{16-19}$ The treatment strategy still remains controversial for normotensive patients with APE, especially if it is submassive $e^{20,21}$ There is a need for a rapid, noninvasive, inexpensive screening test for risk stratification of APE.

Compared with the reported average in-hospital mortality of $8.6 \%, 18,19$ a/APO2 can be used for further risk stratification in normotensive APE. Excluding acute, massive APE with shock, in the present study the 30-day outcome of the remaining 183 patients with normotensive PE were analyzed using the same cut-off value of a/APO2 $<0.49$. This group included small to submassive APE.

The 30-day death result also showed a high NPV of $93.5 \%$ and a PPV of $27.3 \%$. For the 30-day composite event, the PPV was $38.7 \%$, and the NPV was high at $84.4 \%$.

Based on the above analytical results, a/APO2 can help differentiate the high-risk group of APE without hypotension. In daily practice, initial ABG analysis can quickly identify the high-mortality group of normotensive, ensuring that clinicians treat these patients more aggressively with thrombolytic agents.

Kunieda et al have reported that an initially elevated index of gas exchange (AaDO2) would decrease according to recovery of APE, with a linear correlation to the resolved perfusion defect ${ }^{14}$ Moreover, serial a/APO2 analysis may also reveal dynamic change in the severity of APE. However, the initial selection criteria in the present study included either a positive CT scan $(n=81)$ or high-probability lung perfusion scan $(\mathrm{n}=121)$, which resulted in an inconsistent presentation in the extent of PE for these 2 diagnostic tools. Although the a/APO2 supposedly correlates well with the extent of PE, conducting the analysis was limited by the inclusion criteria. A further prospective study is needed to confirm this hypothesis.

\section{Platelets}

Platelet activation occurs in the initial phase of venous thrombus formation. Urinary excretion of thromboxane B2, a marker of platelet activation in the early phases of thrombus formation, increases in APE22 Modig et al reported a reduced platelet count in pulmonary microembolism after trauma $2^{23}$ McCarthy et al has reported that low platelet counts was related to high $\mathrm{AaDO} 2$ values in post-trauma $\mathrm{PE}^{24} \mathrm{In}$ an animal study, significant platelet and fibrinogen consumption occurred after thrombin injection. ${ }^{25}$ Physiological responses to platelet activation include pulmonary hypertension, bronchoconstriction and RV failure.25,26 Thrombocytopenia in APE could be explained by intravascular coagulation and platelet consumption. Further analysis in the present study revealed a relationship between platelet count and a/APO2, suggesting that the degree of thrombocytopenia is related to severity of impaired gas exchange in APE. Moreover, this finding also means that the severity of APE is reflected in the degree of thrombocytopenia.

Linear regression analysis revealed a significant relation- ship between platelet and a/APO2, although the correlation was weak. Two explanations are possible. First, this study analyzed the relationship between a/APO2 and postembolism platelet count, not absolute platelet consumption. Absolute platelet consumption is calculated by pre-embolism platelet count minus post-embolism platelet count. Because the pre- and post-embolism platelet counts vary widely between patients, the severity of APE is more appropriately evaluated by absolute platelet consumption than post-embolism platelet count. Takayoshi et al also demonstrated a strong correlation between physiologic dead space and thromboxane B2 concentration, which was related to the fall in platelet count in an earlier animal study ${ }^{26}$ However, the pre-embolism platelet count is difficult to determine in APE patients, even in a prospective study design.

Second, multiple factors contributed to the final result for impaired gas exchange. The APE causes cardiopulmonary dysfunction that may result from mechanical obstruction, neurogenic factors and local pulmonary vasoconstriction resulting from platelet-mediated release of humoral substances, including serotonin, adenosine diphosphate, prostaglandins, thromboxane and platelet-activating factor 29,30 The correlation between platelet count and a/APO2 would be affected by initial platelet consumption, release of humoral factors, thrombus formation and late-developing events. This impressive linear relationship has never been documented previously.

\section{Hypotension and Short-Term Prognosis of APE}

Some of the parameters identified as predictors of adverse outcome include RV dysfunction, 17-19,21 T-wave inversion,31 elevated troponin level, elevated brain natriuretic peptide 32 hypotension $^{1}$ and abnormal gas exchange 4

Hemodynamic instability has been identified as an important risk factor for in-hospital mortality or escalation of treatment $1,33,34$ The present study also demonstrated the importance of SBP in the short-term prognosis of APE patients. The prevalence of hypotension (SBP $<90 \mathrm{mmHg}$ ) significantly differed between the 30-day survival group and the 30 -day death group ( $<<0.001$, Table 1 ), as well as between the 30-day composite event-free group and the 30day composite event group ( $\mathrm{p}=0.002$, Table 2$)$.

The mechanisms of hypotension depend on the initial mechanical obstruction, as well as on the subsequent neurohumoral stimuli and hypoxic effects ${ }^{29}$ The direct effect of vascular obstruction causes RV dilation and dysfunction, decreasing preload and impairing the gas exchange!11,14,16 Subsequent pulmonary hypertension and ventricular interdependency results in underfilling and decreased end-diastolic volume of the left ventricular. These mechanisms may all cause ischemia and possibly cardiogenic shock.

Massive PE with RV dysfunction and systemic hypotension has a poor prognosis! $1,33,34$ Although the rate of thrombolytic therapy was relatively low in the present study, currently available data indicate that thrombolytic treatment should be performed for hemodynamic instability on presentation?3,34

\section{Study Limitations}

First, this was a retrospective observational study. Thrombolysis was performed by the managing physician based on individual clinical presentation, so the clinical indications of thrombolysis were not uniform. Thus, the parameter "tPA" was not included in the composite endpoint. Further- 
more, predisposing factors were not thoroughly examined in every patient.

Second, there was selection bias in this study. The inclusion criteria allowed enrollment of patients with more severe PE than general APE. According to the analytical results of the Prospective Investigation of Pulmonary Embolism Diagnosis (PIOPED) ${ }^{27} 57 \%$ patients with APE present with low- or intermediate-probability lung perfusion scan. Moreover, Kline et al have reported that approximately $15 \%$ of patients with PE have a normal index of lung gas exchange (AaDO2) ${ }^{28}$ However, in the present study only 3 patients had a normal a/APO2 ratio $(>0.75)$.

Third, the number of subjects was limited. The sample size was not sufficiently large to allow detailed statistical investigation of all different $\mathrm{FiO} 2$ levels. Future studies should analyze a larger study population at every $\mathrm{FiO} 2$ level to further demonstrate the consistency of a/APO2.

Fourth, a previous prospective study proved that the gas exchange index (AaDO2) was valuable for clinically assessing resolved emboli in the recovery phase of APE ${ }^{14}$ In ours retrospective study, not all included patients underwent post-treatment ABG analysis. Subsequent analysis was performed according to clinical presentation of the patients and the decision of the managing physician, which limited the observation of dynamic change in a/APO2 during APE treatment.

\section{Conclusion}

This study has revealed that a/APO2 not only indicates the severity of lung gas exchange, but is also a predictor of in-hospital mortality and composite events. For the purpose of predicting short-term prognosis, a/APO2 is more consistent than $\mathrm{AaDO} 2$ at different $\mathrm{FiO} 2$.

The cut-off value (a/APO2 <0.49) was characterized by a high NPV and moderate PPV in all patients and in the subgroup of normotensive patients with APE. The platelet count presented a linear relationship with severity of a/APO2. a/APO2 is a quick and clinically useful blood test for short-term risk stratification of APE and gives an important insight to the further treatment strategy.

\section{Acknowledgment}

This work was supported by grant CMRPG650031 from Chang Gung Memorial Hospital.

\section{References}

1. Goldhaber SZ, Visani L, De Rosa M. Acute pulmonary embolism: Clinical outcomes in the International Cooperative Pulmonary Embolism Registry (ICOPER). Lancet 1999; 353: 1386-1389.

2. Morio H, Fujimori Y, Terasawa K, Shiga T, Murayama T, Yoshimura $\mathrm{M}$, et al. Pulmonary thromboembolism associated with air travel in Japan. Circ J 2005; 69: 1297-1301.

3. Stein PD, Goldhaber SZ, Henry JW, Miller AC. Arterial blood gas analysis in the assessment of suspected acute pulmonary embolism. Chest 1996; 109: 78-81.

4. Hsu JT, Chu CM, Chang ST, Cheng HW, Cheng NJ, Ho WC, et al. Prognostic role of alveolar-arterial oxygen pressure difference in acute pulmonary embolism. Circ J 2006; 70: 1611-1616.

5. Gilbert R, Kreighley JF. The arterial/alveolar oxygen tension ratio: An index of gas exchange applicable to varying inspired oxygen concentrations. Am Rev Resp Dis 1974; 109: 142-145.

6. Gilbert R, Auchincloss JH Jr, Kuppinger M, Thomas MV. Stability of the arterial/alveolar oxygen partial pressure ratio: Effects of low ventilation/perfusion regions. Crit Care Med 1979; 7: 267-272.

7. Peris LV, Boix JH, Salom JV, Valentin V, Garcia D, Arnau A. Clinical use of the arterial/alveolar oxygen tension ratio. Crit Care Med 1983; 11: 888-891.
8. D'Alonzo GE, Bower JS, DeHart P, Dantzker DR. The mechanisms of abnormal gas exchange in acute massive pulmonary embolism. Am Rev Respir Dis 1983; 128: 170-172.

9. Tapson V. Pulmonary embolism. In: Goldman L, editor. Cecil textbook of medicine, 22nd edn. Philadelphia: Saunders; 2004; 560-561.

10. Huet Y, Lemaire F, Brun-Buisson C, Knaus WA, Teisseire B, Payen D, et al. Hypoxemia in acute pulmonary embolism. Chest 1985; 88: 829-836.

11. Manier G, Castaing Y, Guenard H. Determinants of hypoxemia during the acute phase of pulmonary embolism in humans. Am Rev Respir Dis 1985; 132: 332-338.

12. Kapitan KS, Buchbinder M, Wagner PD, Moser KM. Mechanisms of hypoxemia in chronic thromboembolic pulmonary hypertension. Am Rev Respir Dis 1989; 139: 1149-1154.

13. Levy SE, Simmons DH. Mechanism of arterial hypoxemia following pulmonary thromboembolism in dogs. J Appl Physiol 1975; 39: 41 46.

14. Kunieda T, Okubo S, Fukunaga Y, Naito M, Yoshioka T. Pathophysiologic features of pulmonary thromboembolism in man. Jpn Circ J 1984; 48: 90-99.

15. Gowda MS, Klocke RA. Variability of indices of hypoxemia in adult respiratory distress syndrome. Crit Care Med 1997; 25: 41-45.

16. Torbicki A, Pruszczyk P. The role of echocardiography in suspected and established PE. Semin Vasc Med 2001; 1: 165-174.

17. Ribeiro A. The role of echocardiography Doppler in pulmonary embolism. Echocardiography 1998; 15: 769-778.

18. Grifoni S, Olivotto I, Cecchini P, Pieralli F, Camaiti A, Santoro G, et al. Short-term clinical outcome of patients with acute pulmonary embolism, normal blood pressure, and echocardiographic right ventricular dysfunction. Circulation 2000; 101: 2817-2822.

19. Kasper W, Konstantinides S, Geibel A, Tiede N, Krause T, Just H. Prognostic significance of right ventricular afterload stress detected by echocardiography in patients with clinically suspected pulmonary embolism. Heart 1997; 77: 346-349.

20. Konstantinides S. Should thrombolytic therapy be used in patients with pulmonary embolism? Am J Cardiovasc Drugs 2004; 4: 69-74.

21. Kreit JW. The impact of right ventricular dysfunction on the prognosis and therapy of normotensive patients with pulmonary embolism. Chest 2004; 125: 1539-1545.

22. Klotz TA, Cohn LS, Zipser RD. Urinary excretion of thromboxane B2 in patients with venous thromboembolic disease. Chest 1984; 85: 329-335.

23. Modig J, Hedstrand U, Fischer J, Lundstrom J. Early recognition and treatment of post-traumatic pulmonary microembolism. Crit Care Med 1976; 4: 180-185.

24. McCarthy B, Mammen E, Leblanc LP, Wilson RF. Subclinical fat embolism: A prospective study of 50 patients with extremity fractures. J Trauma 1973; 13: 9-16.

25. Garcia-Szabo R, Johnson A, Malik AB. Thromboxane increases pulmonary vascular resistance and transvascular fluid and protein exchange after pulmonary microembolism. Prostaglandins $1988 ; 35$ : $707-721$.

26. Utsonomiya T, Krausz MM, Levine L, Shepro D, Hechtman HB. Thromboxane mediation of cardiopulmonary effects of embolism. $J$ Clin Invest 1982; 70: $361-368$.

27. The PIOPED Investigators. Value of the ventilation/perfusion scan in acute pulmonary embolism: Results of the prospective investigation of pulmonary embolism diagnosis (PIOPED). JAMA 1990; 263: $2753-2759$

28. Kline JA, Johns KL, Coluciello SA, Israel EG. New diagnostic tests for pulmonary embolism. Ann Emerg Med 2000; 35: 168-180.

29. Stratmann G, Gregory GA. Neurogenic and humoral vasoconstriction in acute pulmonary thromboembolism. Anesth Analg 2003; 97: $341-354$.

30. Rodriguez-Roisin R, Felez MA, Chung KF, Barbera JA, Wagner PD, Cobos A, et al. Platelet-activating factor causes ventilation-perfusion mismatch in humans. J Clin Invest 1994; 93: 188-194.

31. Kosuge M, Kimura K, Ishikawa T, Ebina T, Hibi K, Tsukahara K, et al. Prognostic significance of inverted $\mathrm{T}$ waves in patients with acute pulmonary embolism. Circ J 2006; 70: 750-755.

32. Kucher N, Goldhaber SZ. Cardiac biomarkers for risk stratification of patients with acute pulmonary embolism. Circulation 2003; 108: 2191-2194.

33. Kasper W, Konstantinides S, Geibel A, Olschewski M, Heinrich F, Grosser KD, et al. Management strategies and determinants of outcome in acute major pulmonary embolism: Results of a multicenter registry. J Am Coll Cardiol 1997; 30: 1172-1173.

34. Harris T, Meek S. When should we thrombolyse patients with pulmonary embolism?: A systematic review of the literature. Emerg Med J 2005; 22: 766-771. 